\title{
T-Lymphoblastic Leukemia/Lymphoma and Thymoma: A Case Report and Review of the Literature of a Rare Association
}

\author{
Nadav Mizrahi ${ }^{a, b}$ Eitan Kugler ${ }^{\text {b, c }}$ Lucille Hayman $^{\text {b, } d ~}$ Moshe Yeshurun $^{\text {b, } c}$ \\ Alon Grossman ${ }^{a, b}$ Pia Raananib, c Ofir Wolach ${ }^{b, c}$ \\ aDepartment of Internal Medicine B, Rabin Medical Center - Beilinson Hospital, Rabin Medical Center, Petah-Tikva, \\ Israel; 'bSackler Faculty of Medicine, Tel Aviv University, Tel Aviv, Israel; ' Institute of Hematology, Davidoff Cancer \\ Center, Rabin Medical Center, Petah-Tikva, Israel; ${ }^{\mathrm{d} D e p a r t m e n t}$ of Pathology, Rabin Medical Center - Beilinson \\ Hospital, Rabin Medical Center, Petah-Tikva, Israel
}

\section{Keywords}

Thymoma · T-lymphoblastic lymphoma/leukemia • Leukemia

\section{Abstract}

The co-occurrence of thymoma and T-lymphoblastic lymphoma/leukemia is an extremely rare but previously reported association that poses a diagnostic and therapeutic challenge. We describe a 67-year-old patient with long-standing untreated B1 thymoma that presented with constitutional symptoms and a painless soft tissue mass on the right chest wall. Pathological analysis of the biopsy from the mass demonstrated T-lymphoblastic leukemia/lymphoma. The patient went through a complicated course, was refractory to several lines of therapy, and eventually underwent allogeneic hematopoietic stem cell transplantation in complete remission from a matched related donor. The association between thymoma and malignant neoplasms has been described in the literature, most notably with colorectal adenocarcinoma and thyroid cancer. Thymoma-associated leukemia is, however, extremely unusual, with limited reports in the literature. Distinguishing between thymoma and leukemia can be challenging and often requires meticu- lous diagnostic efforts. For patients with a past history of thymoma, awareness of this particular association should be bared in mind to allow earlier diagnosis and therapy.

(c) 2021 The Author(s).

Published by S. Karger AG, Basel

\section{Introduction}

Thymic neoplasms are a heterogeneous group of tumors and are the most common neoplasms of the anterior mediastinum [1]. Surgery continues to be the mainstay of treatment, and complete resection remains the most important prognostic factor. Association of thymomas with additional neoplasms, most notably colorectal adenocarcinoma and thyroid carcinoma, is well described with a prevalence rate as high as 31\% [2]. Lymphoma occurring in patients with a prior history of a thymoma has also been described in the literature, albeit rarely [3-7]. Despite the well-documented role of the thymus in leukemia genesis in experimental animal models, thymoma is only seldom described to be associated with leukemia, and only anecdotal reports exist of thymoma occurring concomitantly with CML, CLL/SLL, and B-ALL [8-10]. Distinguishing T-lymphoblastic lymphoma/leukemia karger@karger.com www.karger.com/aha

Karger $\stackrel{\text { ' }}{=}$
(C) 2021 The Author(s)

Published by S. Karger AG, Basel

This is an Open Access article licensed under the Creative Commons Attribution-NonCommercial-4.0 International License (CC BY-NC) (http://www.karger.com/Services/OpenAccessLicense), applicable to the online version of the article only. Usage and distribution for commercial purposes requires written permission. 

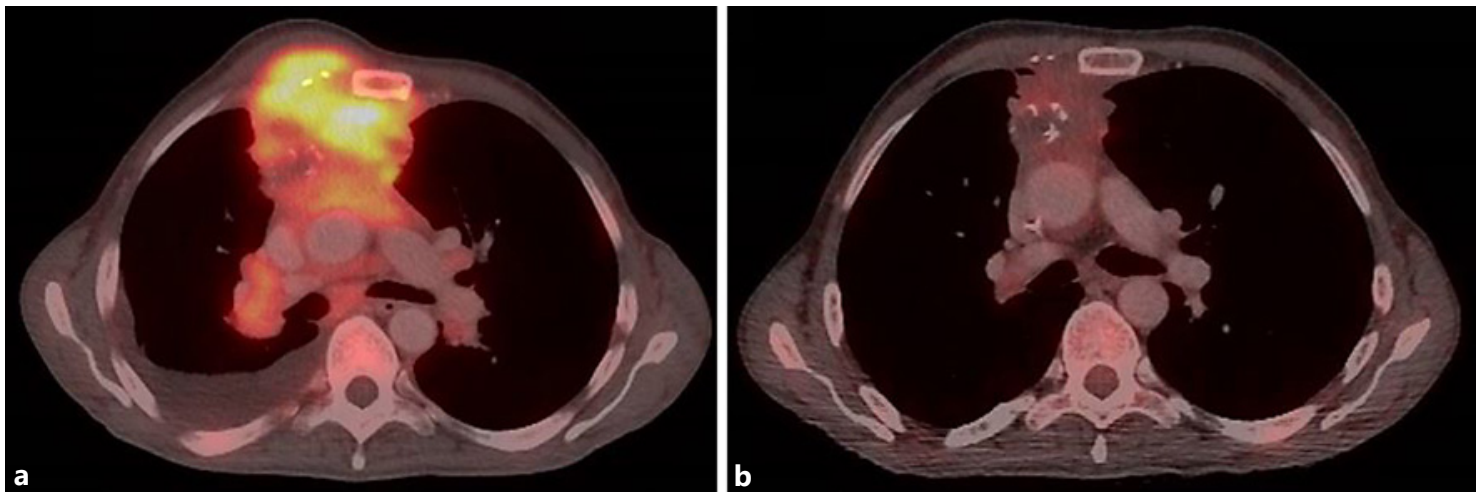

Fig. 1. Comparison of PET-CT scans of the chest demonstrating a right anterior mediastinum large heterogeneous mass invading the chest wall before $(\mathbf{a})$ and after $(\mathbf{b})$ induction therapy with marked reduction in size thereafter.

from thymoma of the B1 type may be particularly challenging as both of these tumors involve pathological lymphocytes that share common morphology and overlapping immunophenotypical markers. In the present report, we describe the diagnosis and management of T-cell lymphoblastic leukemia/lymphoma in a patient with a long-standing untreated $\mathrm{B} 1$ thymoma.

\section{Case Report}

A 67-year-old male admitted to our hospital presented with constitutional symptoms including unintentional weight loss and night sweats, with no systemic fever. The patient reported a mass developing on his right chest wall within the previous few months. The patient's medical history included chronic obstructive pulmonary disease associated with past heavy smoking and type B1 thymoma diagnosed 8 years prior to presentation from an open biopsy of the mediastinum in 2012. The patient declined surgery and was lost to follow-up thereafter. Clinical examination revealed a painless soft tissue mass bulging from the right anterior chest wall along with general lymphadenopathy. Routine blood tests demonstrated mild leukocytosis with monocytosis and a left shift, and predominantly myelocytes and leukoerythroblastic changes were noted in the blood smear. PET-CT scan demonstrated the previously known right anterior mediastinal mass with associated local spreading to the right atrium and a pericardial effusion (Fig. 1). Generalized lymphadenopathy was also noted along with evidence of distant metastatic lesions in the liver, kidneys, and skeleton. Pathological analysis of percutaneous US-guided biopsy of the chest wall mass was compatible with T-lymphoblastic leukemia/ lymphoma, showing sheets of T-cell lymphoblasts positive for CD3, CD5, CD1a, and TdT and complete absence of epithelial cells with negative keratin markers (Fig. 2). Bone marrow (BM) biopsy demonstrated involvement by the same process, with $20 \%$ lymphoblasts of medium size with scarce cytoplasm with abundant typical "hand mirror" morphology. These cells were positive for
CD5, CD7, CD8, CD45, CD1a, and TdT. Flow cytometry (FC) from the marrow demonstrated a similar blast population positive for CD3, CD1a, CD2, CD5, CD7, CD38, CD4, and CD8. Markers for myeloid (e.g., myeloperoxidase) and B-cell lineage were all negative. Cytogenetic analysis showed normal karyotype, with negative fluorescence in situ hybridization analysis for MLL gene rearrangement and $17 \mathrm{p}$ deletion. PCR analysis showed TCR- $\gamma$ monoclonality in tumor tissue. Thus, the diagnosis of T-lymphoblastic leukemia/lymphoma, thymic (cortical) type, was established. Induction therapy according to the modified German Multicenter ALL (GMALL) protocol for elderly patients (dexamethasone, vincristine, daunorubicin, cyclophosphamide, and cytarabine) was initiated after a pre-phase with cyclophosphamide and dexamethasone [11]. At diagnosis, the patient was staged CNS1, though additional intrathecal therapy was withheld due to post-LP subarachnoid hemorrhage during the first induction and was further withheld due to concern of re-bleeding. Interim PET-CT scan and BM aspiration performed after the first induction demonstrated complete remission; however, positive minimal residual disease (MRD) was documented in BM as assessed by PCR. The patient declined allogeneic transplantation at that point and continued asparaginase-based consolidation cycles [12]. Four months into therapy, the patient relapsed with re-growth of the chest mass and lymphadenopathy above and below the diaphragm. He also experienced CNS involvement as assessed by MRI, showing leptomeningeal spread, later confirmed by assessment of the CSF. Repeated BM aspiration failed to demonstrate lymphoid blasts, yet MRD testing remained positive. Repeat biopsy from the protruding mass confirmed disease relapse. A combined salvage approach with systemic therapy with nelarabine and CNS-directed therapy via placement of an Ommaya reservoir was initiated [13]. While CNS disease was cleared, systemic disease persisted, and the patient was salvaged with high-dose cytarabine/mitoxanthrone protocol [14]. Post-salvage was complicated by prolonged aplasia and life-threatening infections, but the patient eventually recovered in complete remission with negative MRD and proceeded to allogeneic hematopoietic stem cell transplantation from a matched related donor. Unfortunately, he relapsed within one and a half months after transplantation and succumbed to his disease. 
Fig. 2. Morphological and immunohistochemical comparison between diagnostic material from the mediastinal mass from the original thymoma diagnosis (2012) and from the later diagnosis of T-lymphoblastic lymphoma/leukemia (2020), depicted by the type of histological stain. $\mathrm{H} \& \mathrm{E}$ stain of the 2020 biopsy showing predominantly medium-sized lymphoblastic-rich infiltrate, highlighted by positive TdT, CD3, and CD1a immunostaining $(\mathbf{d}, \mathbf{f}, \mathbf{j})$. Notice the negative stain for CK5/6 in the T-lymphoblastic lymphoma/leukemia (h) discriminating it from the previous patient's known thymoma (2012) (×20). Scale bars, $100 \mu \mathrm{m}$.

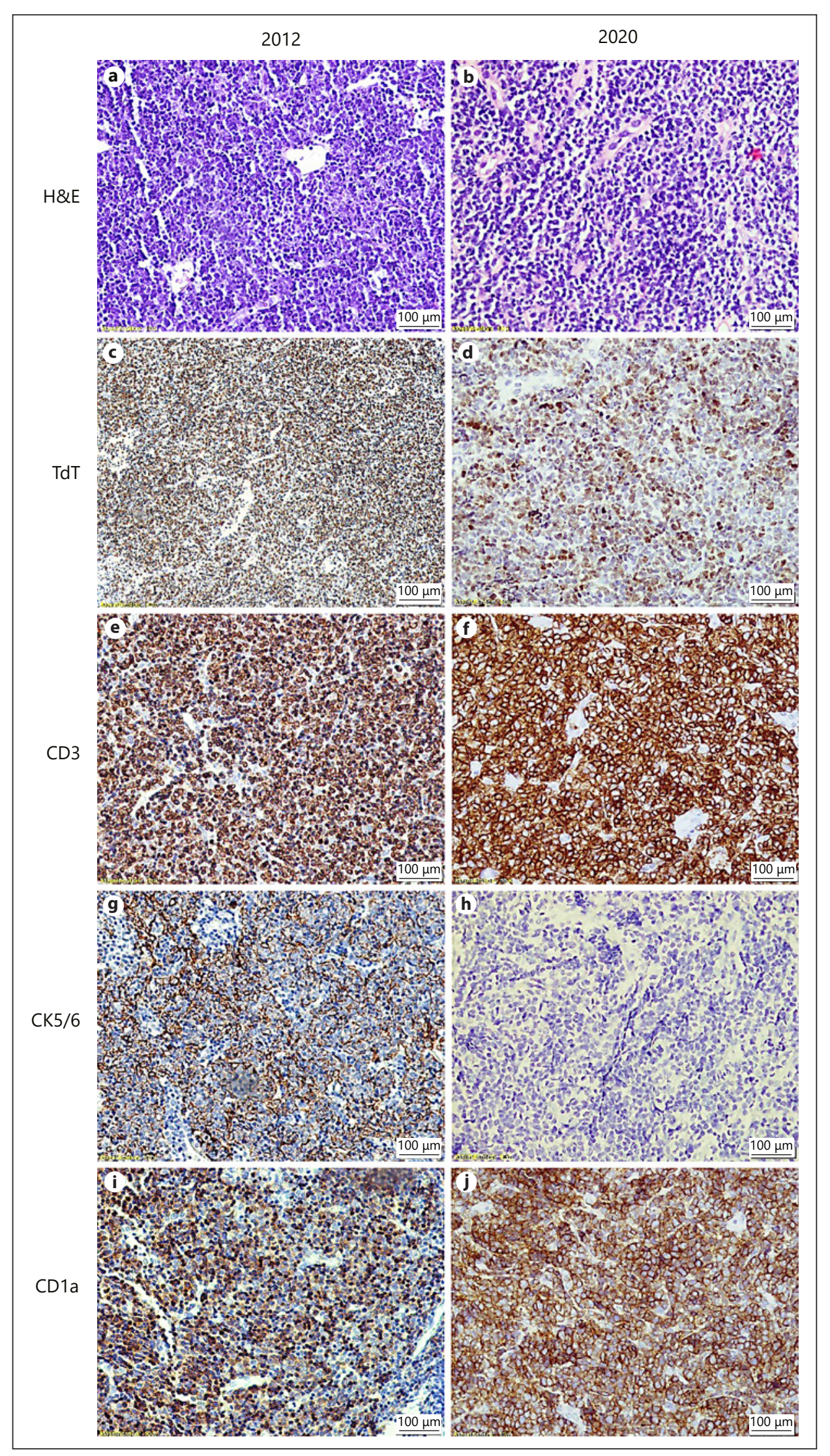

Mizrahi/Kugler/Hayman/Yeshurun/ Grossman/Raanani/Wolach 


\section{Discussion}

Several series have confirmed an increased incidence of thymoma with additional neoplasms of various origins, most notably colorectal adenocarcinoma and thyroid cancer [2]. The precise pathogenic mechanism for this association remains elusive and includes tumorigenesis secondary to radio-chemotherapy treatment for the previous thymoma or susceptibility secondary to perturbations in T-cell immunity after thymus resection. In addition, paraneoplastic conditions typically linked with thymoma, such as myasthenia gravis, pure red cell aplasia, and hypogammaglobulinemia, could conceivably be related to higher rates of cancer either intrinsically or because of immunosuppressive therapy for the associated autoimmune disorder. The patient presented in this case did not have a prior treatment for his thymoma nor any obvious thymoma-associated paraneoplastic immunological phenomena. Moreover, though cytogenetic abnormalities have been reported in thymoma [15], presently no molecular or cytogenetic mechanisms adequately explain the tendency of thymoma patients to be associated with clonal evolution. It can only be speculated that the long-standing untreated thymoma in the present case, with the consequent immunodysregulation or abnormality in T-lymphocyte function, may be in part the basis of the underlying leukemia genesis. The occurrence of leukemia in association with thymoma is rarely described despite the well-documented role of the thymus in leukemogenesis in experimental animal models [810]. Nishioka et al. [16] reported a 43-year-old male patient with myasthenia gravis and invasive thymoma that developed pure red cell aplasia 6 years after radio-chemotherapy and was later diagnosed with T-lymphoblastic lymphoma/leukemia. Boddu et al. [17] published a clinical evaluation for distinguishing thymoma from T-lymphoblastic leukemia/lymphoma based on 4 cases, of which only a single case was diagnosed as T-lymphoblastic lymphoma/leukemia with BM involvement while the other 3 cases were concluded as thymoma with no additional associated hematological malignancy. The above mentioned case was of a man in his mid-40s, originally presented with progressive weight loss with a large mediastinal mass and lymphadenopathy. A left axillary lymph node biopsy and FC was consistent with T-cell lymphoblastic leukemiallymphoma, as confirmed with BM biopsy. In the absence of response after the third chemotherapy cycle, a diagnostic biopsy of the mediastinal mass was performed, and immunohistochemical studies showed the tumor cells to be weakly and focally positive for pan-cytokeratin, consistent with thymoma. The mass was later resected, and the patient was started on salvage therapy for persistent T-lymphoblastic lymphoma/leukemia in BM.

Distinguishing between thymoma and T-lymphoblastic lymphoma/leukemia may be challenging and requires multimodality diagnostic approaches using histological, immunophenotyping, and molecular testing. FC remains an important tool in distinguishing benign thymocytes from T-lymphoblastic lymphoma/leukemia lymphoblasts based on their distinct patterns of antigen expression $[17,18]$. Features favoring thymoma in FC are discrete subpopulations or smearing patterns for CD45 and sCD3, expression of T-cell antigens such as CD2 and CD5 which may be absent in T-lymphoblastic lymphoma/leukemia, and the inhomogeneous pattern of CD4-CD8 coexpression reflecting all stages of normal thymocyte development [17]. Diagnosis can be further challenging in type B1 thymomas, as reported in this case, as these tumors are lymphocyte rich, though low with epithelial component. Thus, detection of keratin-positive neoplastic cells immunohistochemistry may serve as a diagnostic clue [19]. Of note, Ki-67 labeling indices are rarely useful as normally maturing thymocytes in thymoma and TLBL/ALL usually show high Ki-67 expression [17]. Another diagnostic tool is the assessment of characteristic cytogenetic or molecular abnormalities known to be associated with T-lymphoblastic lymphoma/leukemia, such as a monoclonal pattern of TCR- $\beta$ and TCR- $\gamma$ rearrangements by RT-PCR or the characteristic translocations or NOTCH1/FBXW7 mutations which are frequently found in neoplastic Tlymphoblasts as opposed to normal thymocytes [20].

In summary, although thymoma and malignant neoplasms are a well-described association, the association between T-cell leukemia/lymphoma and thymoma is rare but clinically important and challenging. This association may reflect common pathogenetic pathways between these 2 neoplasms. Awareness of this association may allow earlier detection and treatment through proper surveillance.

\section{Statement of Ethics}

This study was approved by the Institutional Review Board (IRB) of the Rabin Medical Center. Patient consent was not applicable since the patient died from his disease, and we could not approach (contact) his next of kin. A waiver for patient consent was approved by the IRB. 


\section{Conflict of Interest Statement}

The authors have no competing interest to declare.

\section{Funding Sources}

No financial support was received.

\section{Author Contributions}

Nadav Mizrahi, Alon Grossman, and Ofir Wolach contributed to conception of the manuscript; Nadav Mizrahi, Eitan Kugler, and Lucille Hayman contributed to literature review; Pia Raanani, Moshe Yeshurun, and Ofir Wolach contributed to supervision and approval of the final version.

\section{Data Availability Statement}

All data generated or analyzed during this study are included in this article. Further enquiries can be directed to the corresponding author.

\section{References}

1 Bushan K, Sharma S, Verma H. A review of thymic tumors. Indian J Surg Oncol. 2013; $4(2): 112-6$.

2 Welsh JS, Wilkins KB, Green R, Bulkley G, Askin F, Diener-West M, et al. Association between thymoma and second neoplasms. JAMA. 2000;283(9):1142-3.

3 Ertel V, Früh M, Guenther A, Cerny T, Fretz C, Cogliatti S. Thymoma with molecularly verified "conversion" to T lymphoblastic leukemia/lymphoma over 9 years. Leuk Lymphoma. 2013;54(12):2765-8.

4 Ito J, Yoshida A, Maeshima AM, Nakagawa K, Watanabe S, Kobayashi Y, et al. Concurrent thymoma, thymic carcinoma, and T lymphoblastic leukemia/lymphoma in an anterior mediastinal mass. Pathol Res Pract. 2015 211(9):693-6.

5 Le Clef Q, Ortega-Sanchez G, Dirnhofer S, Tzankov A. T-lymphoblastic lymphoma after previous thymoma: how NGS helps establishing the diagnosis and procures new insights. Leuk Lymphoma. 2019;60(5):1320-3.

6 Yamato H, Tsutsumi Y, Ehira N, Kanamori $\mathrm{H}$, Shimoyama N, Tanaka J, et al. A case of non-Hodgkin's lymphoma and invasive thymoma treated with R-CHOP therapy. Int Hematol. 2006 Jun;83(5):464-6.

7 Macon WR, Rynalski TH, Swerdlow SH, Cousar JB. T-cell lymphoblastic leukemia/lymphoma presenting in a recurrent thymoma. Mod Pathol. 1991;4(4):524-8.
8 Khoury JD, Amin HM, Jorgensen JL, Ostrowski ML, Bloom MG, Medeiros LJ. Composite thymoma and chronic lymphocytic leukemia/small lymphocytic lymphoma involving the anterior mediastinum. Arch Pathol Lab Med. 2003;127(2):E76-9.

9 Chang H, Chen TJ, Chuang WY, Lin TL. Precursor B-cell acute lymphoblastic leukemia after thymoma and myasthenia gravis: report of a case and review of the literature. Tumori. 2011;97(1):126-9.

10 Knowles DM 2nd. Thymoma and chronic myelogenous leukemia: a case report. Cancer. 1976;38(3):1414-9.

11 Gökbuget N. Treatment of older patients with acute lymphoblastic leukemia. Hematology Am Soc Hematol Educ Program. 2016 Dec; 2016(1):573-9.

12 Egler RA, Ahuja SP, Matloub Y. L-asparaginase in the treatment of patients with acute lymphoblastic leukemia. J Pharmacol Pharmacother. 2016;7(2):62-71.

13 DeAngelo DJ, Yu D, Johnson JL, Coutre SE, Stone RM, Stopeck AT, et al. Nelarabine induces complete remissions in adults with relapsed or refractory T-lineage acute lymphoblastic leukemia or lymphoblastic lymphoma: Cancer and Leukemia Group B study 19801. Blood. 2007 Jun 15;109(12):5136-42.

14 Raanani P, Shpilberg O, Gillis S, Avigdor A, Hardan I, Berkowicz M, et al. Salvage therapy of refractory and relapsed acute leukemia with high dose mitoxantrone and high dose cytarabine. Leuk Res. 1999 Aug;23(8):695700.
15 Goh SG, Lau LC, Sivaswaren C, Chuah KL, Tan PH, Lai D. Pseudodicentric $(16 ; 12)$ (q11; p11.2) in a type $\mathrm{AB}$ (mixed) thymoma. Cancer Genet Cytogenet. 2001;131(1):42-7.

16 Nishioka R, Nakajima S, Morimoto Y, Suzuki H, Nakamura H, Suzuki M. T-cell acute lymphoblastic leukemia with transient pure red cell aplasia associated with myasthenia gravis and invasive thymoma. Intern Med. 1995; 34(2):127-30.

17 Boddu P, Thakral B, Alhuraiji A, Pemmaraju N, Kadia T, Ohanian M, et al. Distinguishing thymoma from T-lymphoblastic leukaemia/ lymphoma: a case-based evaluation. J Clin Pathol. 2019;72(3):251-7.

18 Li S, Juco J, Mann KP, Holden JT. Flow cytometry in the differential diagnosis of lymphocyte-rich thymoma from precursor T-cell acute lymphoblastic leukemia/lymphoblastic lymphoma. Am J Clin Pathol. 2004;121(2): 268-74.

19 Adam P, Hakroush S, Hofmann I, Reidenbach S, Marx A, Ströbel P. Thymoma with loss of keratin expression (and giant cells): a potential diagnostic pitfall. Virchows Arch. 2014;465(3):313-20.

20 Van Vlierberghe P, Ferrando A. The molecular basis of T cell acute lymphoblastic leukemia. J Clin Invest. 2012;122:3398-406. 